\title{
Overexpression of Regulatory $T$ Cell-Related Markers (FOXP3, CTLA-4 and GITR) by Peripheral Blood Mononuclear Cells from Patients with Breast Cancer
}

\author{
Esmat Khalife ${ }^{1,2}$, Ali Khodadadi ${ }^{3}$, Abdolhosein Talaeizadeh ${ }^{4}$, Leila Rahimian ${ }^{1}$, \\ Maryam Nemati ${ }^{5}$, Abdollah Jafarzadeh ${ }^{2,6 *}$
}

\begin{abstract}
Background: Regulatory T (Treg) cells are immunosuppressor lymphocytes that play a critical role in the establishment and progression of cancers. A number of markers, especially FOXP3, CTLA-4 and GITR influence the function of Treg cells. This investigation aimed to evaluate the expression of a number of important Treg cell-related markers by peripheral blood mononuclear cells (PBMCs) from newly-diagnosed women with breast cancer. Methods: The fresh PBMCs were obtained from 20 women with breast cancer and 20 healthy individuals. The PBMCs from both groups were cultured for 32 hours in the presence or absence of PHA $(10 \mu \mathrm{g} / \mathrm{ml})$. After total RNA extraction from cultured PBMCs, the expression of the FOXP3, CTLA-4 and GITR transcripts was assessed using real time-PCR. Results: The mRNA expression of FOXP3, CTLA-4 and GITR in unstimulated PBMCs from patients with breast cancer were significantly higher than healthy control group $(\mathrm{P}<0.05, \mathrm{P}<0.03$ and $\mathrm{P}<0.04$, respectively). Similarly, the expression of FOXP3, CTLA-4 and GITR transcripts in PHA-stimulated PBMCs from patients with breast cancer were significantly increased in comparison with healthy individuals $(\mathrm{P}<0.01, \mathrm{P}<0.005$ and $\mathrm{P}<0.01$, respectively). Conclusion: The increased expression of FOXP3, CTLA-4 and GITR represent higher activity of Treg cells in patients with breast cancer that may play an important role in the tumor establishment and development.
\end{abstract}

Keywords: Breast cancer- regulatory T cells- FOXP3- CTLA-4- GITR

Asian Pac J Cancer Prev, 19 (11), 3019-3025

\section{Introduction}

Breast cancer is expected to account for $29 \%$ of all newly diagnosed cancers and $15 \%$ of the all cancer-related deaths among women, worldwide (Siegel et al., 2016). The immune system plays an essential role in defense against tumor cells so that the patients with suppressed or compromised immune function have a very increased incidence of malignancy (Casey et al., 2014). Based on the immune surveillance concept, one of the major duty of the immune system is to identify and kill cancerous cells as they appear (Monzavi-Karbassi et al., 2013). The elements of the both innate and adaptive immunity, such as natural killer (NK) cells, NKT cells, macrophages, neutrophils, eosinophils, specific cytotoxic T lymphocytes (CTLs), antibodies and some cytokines exhibit antitumor activity (Casey et al., 2014; Monzavi-Karbassi et al., 2013).

However, the tumor cells escape from immune recognition/killing by several mechanisms, especially down-regulating of immune responses (Spranger, 2016). Some abnormalities in immune-related parameters were observed in patients with breast cancer (Jafarzadeh et al., 2015a; Jafarzadeh et al., 2015b; Jafarzadeh et al., 2016). The purpose of cancer immunotherapy is to robust the immune system to recognize and kill tumor cells by overwhelming the pathways by which tumor cells evade and suppress the immune responses (Sheikhi et al., 2016).

$\mathrm{CD}^{+} \mathrm{T}$ helper $(\mathrm{Th})$ cells play multiple functions in the induction of immune responses against tumor cells. The effector $\mathrm{CD}^{+} \mathrm{T}$ cells are classified into diverse subsets including Th1, Th2, Th17 and regulatory T (Treg) lymphocytes based on the synthesis of a specific cytokine profile (Golubovskaya and $\mathrm{Wu}, 2016$ ). Th1 cells produce cytokines such as IFN- $\gamma$, IL-2, IL-12 and tumor necrosis factor-beta (TNF- $\beta$ ) that exhibit strong anti-tumor activities by activating $\mathrm{CD} 8^{+}$CTLs and NK-mediated cytotoxicity, as well as improving the expression of major

${ }^{1}$ Department of Immunology, School of Medicine, ${ }^{2}$ Molecular Medicine Research Center, Research Institute of Basic Medical Sciences Rafsanjan University of Medical Sciences, Rafsanjan, ${ }^{3}$ Department of Immunology, ${ }^{4}$ Department of Surgery, School of Medicine, Ahvaz Jundishapur University of Medical Sciences, Ahvaz, ${ }^{5}$ Department of Laboratory Sciences, ParaMedical School, ${ }^{6}$ Department of Immunology, School of Medicine, Kerman University of Medical Sciences, Kerman, Iran. *For Correspondence: Jafarzadeh14@yahoo.com 
histocompatibility complex (MHC) and costimulatory molecules on the surface of antigen presenting cells (APCs) (Golubovskaya and $\mathrm{Wu}, 2016$; Kursunel and Esendagli, 2016). Conversely, Th2 cell-related cytokines (including IL-4, IL-5, IL-6 and IL-13) inhibit anti-tumor immune responses by down-regulation of Th1 cells (Golubovskaya and $\mathrm{Wu}, 2016$; Jafarzadeh et al., 2015b). A large number of pro-inflammatory cytokines release by Th17 cells, particularly IL-17 (also known as IL-17A), IL-17F, IL-21, IL-22 and GM-CSF (Etesam et al., 2016; Guery and Hugues, 2015). There are some controversies and inconsistencies regarding the role of Th17 cells in tumor immunology. The proor anti-tumor effects of Th17 cells may be exert in a tumor type-dependent manner. Therefore, Th17 cell-associated immune responses were related with both good or bad prognoses in cancer investigations (Guery and Hugues, 2015).

Regulatory $\mathrm{T}$ (Treg) cells play a prominent role in the regulation of the immune activities and maintain tolerance to self-antigens through a number of mechanisms such as suppression of antigen-presenting cells via CTLA-4, secretion of immunomodulatory cytokines (IL-10, TGF- $\beta$ and IL-35), expression of granzyme/perforin, consumption of IL-2, and degradation of ATP (Jafarzadeh et al., 2015c; Takeuchi and Nishikawa, 2016). Treg cells make up $5-15 \%$ of the $\mathrm{CD}^{+} \mathrm{T}$ cells and there are two subsets of Treg cells, including natural Treg (nTreg) cells that develop in the thymus and inducible Treg (iTreg) cells that arise from naïve $\mathrm{CD} 4^{+} \mathrm{T}$ cells in the peripheral tissues after antigenic stimulation, in the presence of TGF- $\beta$ and IL-2, respectively (Jafarzadeh et al., 2015c; Noack and Miossec, 2014). The differentiation, maintenance and suppressor function of Treg cells is regulated by a master transcription factor Forkhead Box P3 (FOXP3) which is encoded by a related gene that mapped on the $\mathrm{X}$ chromosome (Etesam et al., 2016; Jafarzadeh et al., 2015c; Szylberg et al., 2016).

Treg cells suppress a wide range of immune cells such as $\mathrm{CD}^{+} \mathrm{T}, \mathrm{NK}, \mathrm{B}$ and APCs (Jafarzadeh et al., 2015a; Muenst et al., 2016). Treg cells are aggregated into tumor tissues by chemokine CCL22 that is secreted by tumor cells and macrophages (Jafarzadeh et al., 2015a; Muenst et al., 2016). There are some investigations regarding the Treg cell-dependent immunosuppression in cancer patients (Hatanaka et al., 2014; Tohyama et al., 2013). The suppression of cell-mediated immunity and the enhancing of the number and/or function of Treg cells may lead to the tumor progression (Hatanaka et al., 2014). The immunosuppressive functions of Treg cells are influenced by some markers, particularly FOXP3, Cytotoxic $\mathrm{T}$ lymphocyte antigen-4 (CTLA4) and glucocorticoid induced TNFR family-related gene (GITR) (Nasser et al., 2014; Rathod et al., 2014).

The $\mathrm{CD} 4^{+} \mathrm{T}$ cells recognizing MHC-antigenic peptide which is presented on the surface of APCs (Beyersdorf et al., 2015). The recognition of MHC-antigenic peptide by $\mathrm{T}$ cell receptor (TCR) is necessary for T cell activation. In addition, the binding of costimulatory molecules, especially CD28, on the T cell to counterpart molecules B7-1 and B7-2 on the APCs providing a second costimulatory signal which is required for full activation of T cell (Beyersdorf et al., 2015; Buchbinder and Hodi, 2015). Since B7-1 and B7-2 molecules bind to both CTLA- 4 and CD28 molecules, therefore, CTLA-4 may negatively influences $\mathrm{T}$ cell activation by competing with CD28 for attachment to B7 molecules. CTLA-4 is also constitutively expressed on Treg cells and its ligation positively reinforcements the immunosuppressive functions of Treg cells (Buchbinder and Hodi, 2015).

The GITR is also constitutively expressed at high amounts on the surface of Treg cells, but at low quantities on naïve and memory T lymphocytes (Knee et al., 2016). Following TCR engagement, the GITR ligation on T cells with its ligand (GITRL) or agonist antibodies, reinforces $\mathrm{T}$ cell activation by inducing IL- 2 and IFN- $\gamma$ expression, enhancing CD25 expression and promoting cell expansion (Knee et al., 2016). Although, high expression of GITR is a feature for Treg cells, its function on these cells is more complex. There are some reports showing that GITR ligation inhibits immunosuppressive activities of Treg cells (Knee et al., 2016). In a murine model it has been indicted that GITR acts as a costimulatory molecule for activation of Treg cells (Bianchini et al., 2011).

Although, there are a number of reports regarding the determination of the FOXP 3 and CTLA- 4 expression in the peripheral blood from patients with breast cancer (Jaberipour et al., 2010; Kawaguchi et al., 2017), however, there is no investigation regarding the measurement of FOXP3, CTLA-4 and GITR in PBMCs from malignant patients after in vitro stimulation. This study aimed to evaluate the expression of some Treg cell related markers (including FOXP3, CTLA4 and GITR) by PBMCs from patients with newly diagnosed breast cancer following the activation with phytohemagglutinin (PHA) mitogen.

\section{Materials and Methods}

\section{Subjects}

From October 2015 up to May 2016, 20 women with breast cancer (mean age: $41.25 \pm 9.07$ years) were selected among patients who referred to the affiliated hospitals of Jundishapur University of Medical Sciences. The patients were newly diagnosed, and enrolled to study before they receive chemotherapy, radiotherapy or immunotherapy. The presence of breast cancer and its staging was confirmed by expert oncologists based on the defined surgical and pathological observations and also according to the criteria of the Sixth Edition of the American Joint Committee on Cancer (AJCC) (Singletary et al., 2003). Moreover, 20 healthy women (mean age: $39.45 \pm 7.89$ years) were enrolled into the study as a control group. The healthy individuals were randomly recruited among blood donors and were in good health, without any acute or chronic illnesses. The procedure was evaluated and approved by the Ethical Committees of Rafsanjan University of Medical Sciences and the written informed consent was also obtained from participants before blood sampling.

Isolation of peripheral blood mononuclear cells (PBMCs) Five to ten $\mathrm{mL}$ of fresh peripheral blood was collected 
into heparinized tubes from healthy women and patients with breast cancer. The PBMCs were separated by a standard procedure using Ficoll-Paque (Sigma, USA). Briefly, a 1:2 dilution was made by adding an equal volume of phosphate buffered saline (PBS) to a blood sample. The diluted blood was then carefully layered over the equal volume of Ficoll-Paque solution. The sample was centrifuged for 30 minutes at $20^{\circ} \mathrm{C}$, with a rate of $3000 \mathrm{rpm}$. Immediately after centrifugation, the upper layer was removed and discarded. The mononuclear cell layer was carefully transferred to a new $15 \mathrm{~mL}$ tube and washed three times by mixing with $10 \mathrm{~mL}$ of Roswell Park Memorial Institute (RPMI)-1640 media. Then, the final pellet of PBMCs were resuspended in a complete culture media of RPMI-1640 containing 10\% heat inactivated fetal bovine serum (Gibco Life Techologies Ltd, Paisley, $\mathrm{UK})$ and antibiotics, including penicillin $(100 \mathrm{U} / \mathrm{ml})$ and streptomycin $(100 \mu \mathrm{g} / \mathrm{ml})$. The cells were then seeded at $1 \times 10^{6}$ cells per milliliter in 24-well culture plates (Nunc, Thermo Fisher Scientific Inc, Denmark) in the presence or absence of $10 \mu \mathrm{g} / \mathrm{ml}$ of a $\mathrm{T}$ cell stimulator, phytohemagglutinin [(PHA) (Gibco Life Techologies Ltd, Paisley, UK)]. The PBMCs were incubated for 32 hours at $37^{\circ} \mathrm{C}$ in a $5 \% \mathrm{CO}_{2}$ incubator. After 32 hours, the cells were harvested for total RNA extraction and further experiments.

$R N A$ extraction, reverse transcription and quantitative real-time $P C R$

A Trizol reagent (Bionner, Korea) was used to extract the total RNA from the PBMCs, according to the manufacturer's guideline. The quality of the extracted RNA was determine by electrophoresis on the agarose gel pre-treated with ethidium bromide. A spectrophotometer system used to measure the purity and quantity of RNA, basis on the measuring its absorption at $260 \mathrm{~nm}$ and $280 \mathrm{~nm}$. A complementary DNA (cDNA) synthesis kit (Applied Biosystems, USA) was used to generate cDNA from extracted RNA. The reverse transcription protocol was included: $70^{\circ} \mathrm{C}$ for 10 minutes (in the absence of reverse transcription enzyme), $20^{\circ} \mathrm{C}$ for 1 minute (cooling stage), addition of reverse transcription enzyme, $42^{\circ} \mathrm{C}$ for 60 minutes, and eventually the protocol was completed by a step at $95^{\circ} \mathrm{C}$ for 10 minutes to halt the function of the reverse transcription enzyme.

The analysis of the gene expression of FOXP3, CTLA4 and GITR was performed using a real-time PCR instrument (Applied Biosystems, USA) using a SYBR green master mix (Applied Biosystems, USA), mixed with $200 \mathrm{ng}$ of template cDNA with $2 \mu \mathrm{L}$ suitable primers (10 pmol stock). The primers synthesized by Bioneer Company (Korea). The sequences of the used primers were indicated in Table 1. The program of thermal cycler was entailed: $50^{\circ} \mathrm{C}$ for 2 minutes, $95^{\circ} \mathrm{C}$ for 2 minutes; 40 cycles of $95^{\circ} \mathrm{C}$ for 15 seconds, $60^{\circ} \mathrm{C}$ for 1 minutes, $95^{\circ} \mathrm{C}$ for 15 seconds.

For normalization of the amplified target genes, the GAPDH gene was used as an internal control or housekeeping gene. The amount of the FOXP3, CTLA4 and GITR expression in the PBMCs expressed as units relative to the amount of GAPDH expression that was calculated by the $2^{-\Delta \mathrm{CCt}}$ formula. The PCR products were also electrophoresed and observed on a $1 \%$ agarose gel comprising $0.5 \mathrm{mg} / \mathrm{ml}$ ethidium bromide.

\section{Statistical analysis}

The data were presented as mean \pm SEM. Statistical comparison was analyzed by a computer program (SPSS version 18, Chicago, IL, USA) by using ANOVA and Student's t test, as appropriate. The P values less than 0.05 were considered to be statistically significant.

\section{Results}

The expression of FOXP3 by PBMCs from breast cancer patients and healthy group

The fold change of the gene expression of FOXP3 in the unstimulated and PHA-stimulated PBMC from women with breast cancer and healthy subjects have been demonstrated in Table 2 and Figure 1 . In healthy women, the expression of FOXP3 mRNA in PHA-stimulated cultures was significantly lower compared with unstimulated cultures $(\mathrm{P}<0.02)$. In patients with breast cancer, the amounts of the FOXP3 expression in the PHA-stimulated PBMCs was also lower than unstimulated cultures, but the difference did not reach to a significant level. The amounts of the FOXP3 expression were significantly increased in the unstimulated and PHA-stimulated PBMCs from patients with breast cancer compared with equal cultures from healthy women $(\mathrm{P}<0.05$ and $\mathrm{P}<0.01$, respectively).

\section{The expression of CTLA4 by PBMCs from breast cancer} patients and healthy group

In both healthy normal and breast cancer groups, the amounts of the CTLA4 mRNA expression in cultures stimulated with PHA was significantly higher than unstimulated PBMCs ( $\mathrm{P}<0.001$ and $\mathrm{P}<0.01$, respectively) (Figure 2 and Table 2). The amounts of the CTLA4 expression by unstimulated and PHA-induced PBMCs from patients with breast cancer were significantly higher than counterpart cultures from healthy women $(\mathrm{P}<0.03$ and $\mathrm{P}<0.005$, respectively) (Figure 2 and Table 2).

Table 1. The Used Primers for the Assessment of the mRNA Expression of FOXP3, CTLA4 and GITR by PBMCs from Healthy Women and Breast Cancer Patients

\begin{tabular}{|c|c|}
\hline Genes & Primers \\
\hline \multirow[t]{2}{*}{ FOXP3 } & Forward: 5-GTGGCCCGGATGTGAGAAG-3 \\
\hline & Reverse: 5-GGAGCCCTTGTCGGATGATG-3 \\
\hline \multirow[t]{2}{*}{ CTLA4 } & Forward: 5-GCCCTGCACTCTCCTGTTTTT-3 \\
\hline & Reverse: 5-GGTTGCCGCACAGACTTCA-3 \\
\hline \multirow[t]{2}{*}{ GITR } & Forward: 5-GAGTGGGACTGCATGTGTGT-3 \\
\hline & Reverse: 5-ACTGAATTTCCCCTGGGACT-3 \\
\hline \multirow[t]{2}{*}{ GAPDH } & Forward: 5-TTCCAATATGATTCCACCCA-3 \\
\hline & Reverse: 5-GATCTCGCTCCTGGAAGATG-3 \\
\hline
\end{tabular}




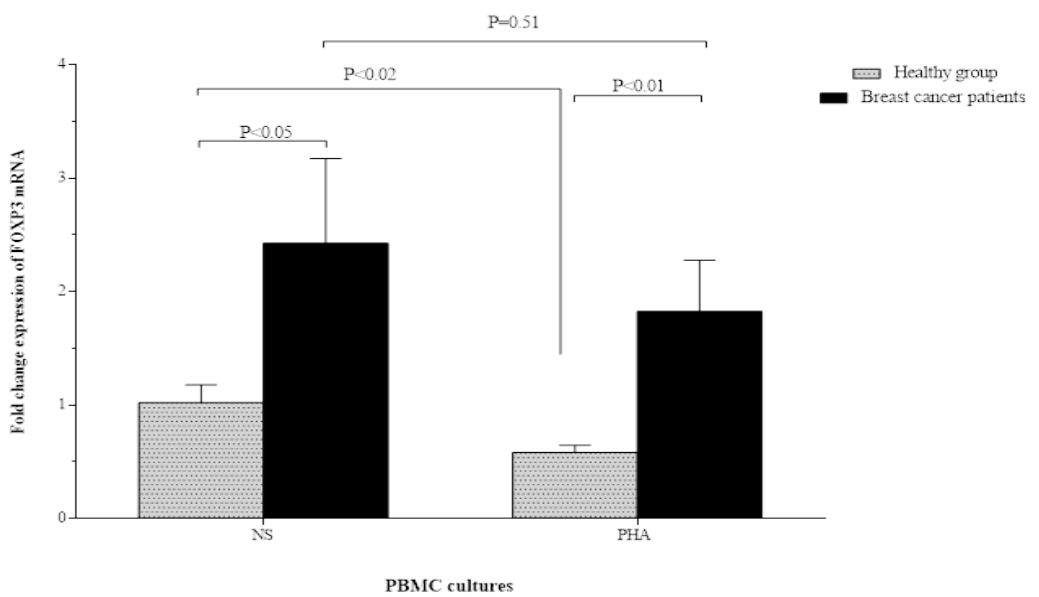

Figure 1. The mRNA Expression of FOXP3 by Unstimulated and PHA-stimulated PBMCs from the Healthy Women and Breast Cancer Patients. The FOXP3 expression by unstimulated and PHA-stimulated PBMCs from patients with breast cancer was significantly higher than equal cultures from healthy women.

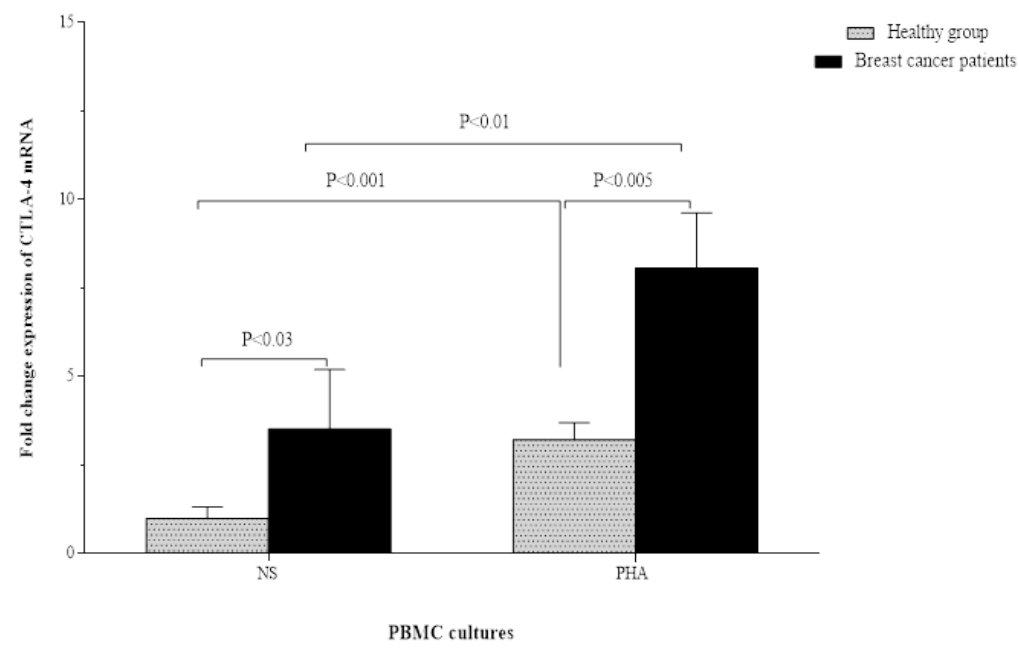

Figure 2. The mRNA Expression of CTLA-4 by Unstimulated and PHA-stimulated PBMCs from the Healthy Women and Breast Cancer Patients. The CTLA4 expression by unstimulated and PHA-stimulated PBMCs from patients with breast cancer significantly was higher than equal cultures from healthy women.

The expression of GITR by PBMCs from breast cancer patients and healthy group

In both normal control and breast cancer groups, the PHA-induced expression of GITR mRNA was lower than unstimulated PBMCs, but the differences were not significant (Figure 3 and Table 2). The fold change expression of GITR by unstimulated and PHA-stimulated PBMCs from patients with breast cancer were significantly increased as compared with equal cultures from healthy subjects $(\mathrm{P}<0.04$ and $\mathrm{P}<0.01$, respectively) (Figure 3 and Table 2 ).

\section{Discussion}

Results from our study showed that the mRNA expression of FOXP3 in PBMCs stimulated with PHA was significantly decreased as compared with unstimulated cultures. In both healthy control and breast cancer groups, the PHA-induced CTLA4 expression was significantly higher than unstimulated PBMCs. However, the amounts of the GITR mRNA expression did not significantly differ between unstimulated and stimulated PBMCs neither in the healthy women nor in patients with breast cancer. These findings represent that PHA may differentially influence the expression of

Table 2. The Expression of FOXP3, CTLA4 and GITR by PBMC from Healthy Women and Breast Cancer Patients

\begin{tabular}{lcccc}
\hline Groups & Stimulator of PBMCs & FOXP3 expression & CTLA4 expression & GITR expression \\
\hline Healthy group & Without stimulation & $1.02 \pm 0.15$ & $1.00 \pm 0.32$ & $1.01 \pm 0.41$ \\
& PHA & $0.58 \pm 0.06$ & $3.22 \pm 0.47$ & $0.60 \pm 0.16$ \\
Breast cancer group & Without stimulation & $2.42 \pm 0.74$ & $3.52 \pm 1.67$ & $4.92 \pm 1.68$ \\
& PHA & $1.82 \pm 0.45$ & $8.07 \pm 1.54$ & $3.37 \pm 1.01$ \\
\hline
\end{tabular}




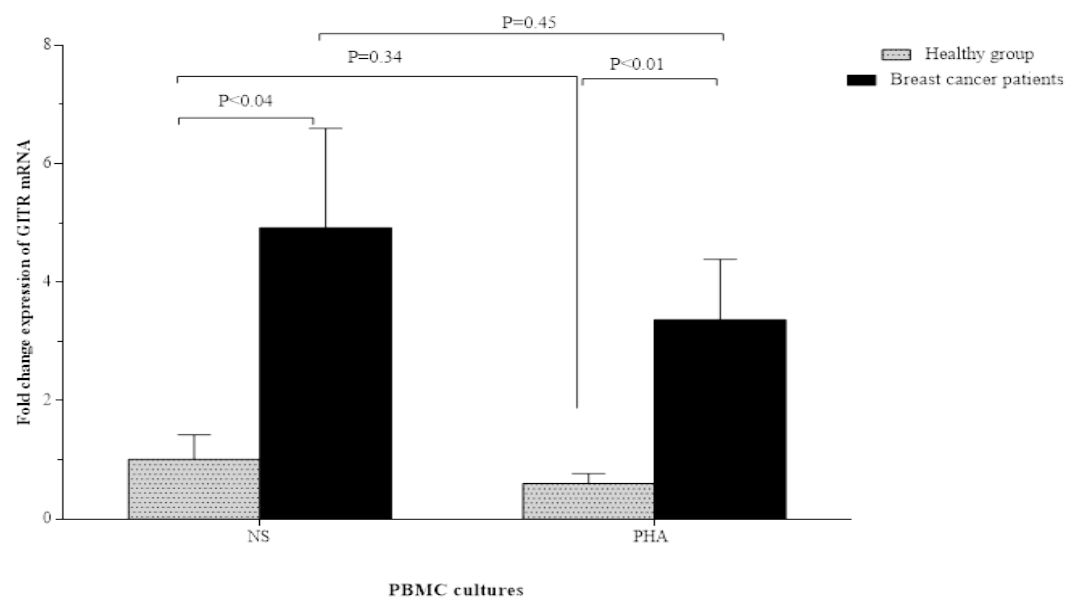

Figure 3. The mRNA Expression of GITR by Unstimulated and PHA-stimulated PBMCs from the Healthy Women and Breast Cancer Patients. The GITR expression by unstimulated and PHA-stimulated PBMCs from patients with breast cancer was significantly higher than equal cultures from healthy women.

the FOXP3, CTLA-4 and GITR. In agreement with our results, it has been reported that $\mathrm{PHA}$-induced expression of FOXP3 was reduced in healthy subjects and patients with atherosclerosis (Mottaghi et al., 2012). The influences exerted by PHA may be contributed to the polyclonal activation of $\mathrm{T}$ cells, which lead to the differentiation of naïve $\mathrm{T} \mathrm{CD} 4^{+}$into various effector $\mathrm{T}$ cells, including Th1-, Th2- and Th17 cells, and these aforementioned cells may have inhibitory effects on the FOXP3 expression and Treg cell differentiation. In patients with breast cancer, the amounts of the FOXP3 expression in PBMCs stimulated with PHA was lower than unstimulated cells, but the difference was not statistically significant. This difference may be due to the higher frequency of Treg cells in patients with breast cancer in comparison with healthy individuals.

The results of the present investigation indicated that the gene expression of a number of Treg cell-related markers including FOXP3, CTLA4 and GITR were significantly increased in both unstimulated- and PHA-treated PBMCs from newly-diagnosed patients with breast cancer in comparison with healthy control women. The increased expression of FOXP3 lead to an increase in the number of Treg cells and enhanced immunosuppressive function in patients with breast cancer. Moreover, the elevated amounts of the FOXP3 expression by PHA-treated PBMCs from patients with breast cancer representing an increased potential for differentiation of Treg cells that lead to the suppression of anti-tumor immunity.

The results of this study also indicated that the gene expression of CTLA4 was significantly increased in both unstimulated- and stimulated PBMCs from patients with breast cancer as compared with healthy control group. CTLA-4 palys an important role in damping of anti-tumor immunity and therefore in cancer development and progression (Buchbinder and Hodi, 2015). Increased number of Treg cells and higher expression of CTLA4 have been observed in patients with various types of cancer compared to control individuals (Kordi-Tamandani et al., 2014). Binding of CTLA-4 on $\mathrm{T}$ cells to B7 molecules on APCs down-regulates the activation of T-cells through various mechanisms (Beyersdorf et al., 2015). Since B7 molecules bind to both CTLA-4 and CD28 molecules, CTLA-4 may competitively block CD28-B7 interactions. Moreover, CTLA-4 can directly suppress the TCR-mediated signaling, decrease the expression of IL-2 and its receptor, and modulate the cell proliferation (Buchbinder and Hodi, 2015). It has been also reported that CTLA-4 may contribute in the generation of peripheral tolerance through induction of apoptosis or anergy in antigen specific T cells (Buchbinder and Hodi, 2015). It has been demonstrated that the CTLA-4-blocking antibodies increase antitumor immune responses through promotion of the CD8 ${ }^{+} \mathrm{CTL}$ activation and depletion of Treg cells (Buchbinder and Hodi, 2015). As mentioned the CTLA-4 is constitutively expressed on Treg cells and antibodies against CTLA-4 lead to the depletion of intratumoral Treg cells, perhaps via induction of antibody-dependent cell-mediated cytotoxicity (ADCC) (Buchbinder and Hodi, 2015).

Here, we also showed for the first time that the expression of GITR by both unstimulated and stimulated PBMCs from patients with breast cancer was higher than healthy control group. There are some reports regarding the damping of anti-tumor immunity by GITR. It has been demonstrated in patients with acute myeloid leukemia (ALL) that the GITR ligation on NK cells impair NK cytotoxicity, reduce IFN- $\gamma$ production and enhance IL-10 secretion (Baessler et al., 2009). IFN- $\gamma$ plays an important role in the limiting of malignant cells as it has anti-proliferative, anti-angiogenic and pro-apoptotic effects (Kursunel and Esendagli, 2016). IFN- $\gamma$ also enhances antigen presentation to $\mathrm{T}$ cells, stimulates the synthesis of the tumoricidal intermediates such as reactive oxygen species (ROS) and nitric oxide (NO), increases the expression of IL-12, IL-18, and CD86 by APCs, promotes the differentiation of Th1 cells, potentiates the CTL function, and inhibits Th2 and Treg cell differentiation (Kursunel and Esendagli, 2016). On the other hand, IL-10 may help tumor cell to escape from immune system through damping the anti-tumor immune response (Mannino et al., 2015). It has been indicated that the blocking of GITR on NK cells enhances their cytotoxicity 
and IFN- $\gamma$ secretion (Baessler et al., 2009).

GITR marker was also expressed at high amounts on the surface of NK cells from patients with Chronic lymphocytic leukemia (CLL) (Buechele et al., 2012). Upon interaction of GITR with GITRL on leukemia cells, the GITRL-mediated signaling lead to the secretion of IL-6, IL- 8 and TNF- $\alpha$, which act as proliferative and survival factors for cancer cells (Buechele et al., 2012). In addition, GITR ligation impair the NK activity and reduce the IFN- $\gamma$ production by NK cells, which could be improve by GITR blocking antibodies. Thus, GITR-GITRL interaction may involve in the pathogenesis of CLL and inhibit the NK cell-mediated tumor cell killing (Buechele et al., 2012).

On the other hand, the results of a number of investigations in murine model were indicated the participation of GITR in the induction of antitumor immune responses. For example, the administration of agonistic antibodies against GITR enhances survival rates in tumor-bearing animals (Naidoo et al., 2014). In addition, the combined programmed Death 1 (PD-1) blockade and GITR stimulation induce a powerful antitumor immunity in a murine ovarian cancer model (Lu et al., 2014). In the murine models, the anti-tumor activity of GIRT has been mainly attributed to the stimulation of CTLs and Th1 cells, induction of M1 macrophages and inhibition of Treg cells (Kawano et al., 2015; Patel et al., 2016). Therefore, it seems that GITR may plays different roles in mice and humans (Nocentini et al., 2012). Furthermore, species-related differences concerning the effects of GITR on the T cells have also been indicated (Nocentini et al., 2012). In contrast to the murine system, GITR does not inhibit human Treg cells-related immunosuppression (Levings et al., 2002). It should be also noted that GITR role in the anti-tumor immunity may be exert in a tumor type-dependent manner. Further investigations are required to clearly elucidate whether GITR mediates various influences in different tumors.

In conclusion, the higher expression of FOXP3, CTLA4 and GITR represent higher activity of Treg cells in patients with breast cancer that may play a role in the tumor development. These findings emphasize that the targeting of Treg cells with aforementioned markers may be a suitable strategy for breast cancer immunotherapy.

\section{Conflict of interest}

The authors have no any conflict of interest.

\section{Acknowledgments}

This work was funded by a grant from Rafsanjan University of Medical Sciences, Rafsanjan, Iran.

\section{References}

Baessler T, Krusch M, Schmiedel BJ, et al (2009). Glucocorticoid-induced tumor necrosis factor receptor-related protein ligand subverts immunosurveillance of acute myeloid leukemia in humans. Cancer Res, 69 , 1037-45.

Beyersdorf N, Kerkau T, Hunig T (2015). CD28 co-stimulation in T-cell homeostasis: a recent perspective. Immunotargets Ther, 4, 111-22.

Bianchini R, Bistoni O, Alunno A, et al (2011). CD4 ${ }^{+}$ CD25lowGITR+ cells: A novel human $\mathrm{CD}^{+} \mathrm{T}$-cell population with regulatory activity. Eur J Immunol, 41, 2269-78.

Buchbinder E, Hodi FS (2015). Cytotoxic T lymphocyte antigen-4 and immune checkpoint blockade. J Clin Invest, 125, 3377-83.

Buechele C, Baessler T, Wirths S, et al (2012). Glucocorticoid-induced TNFR-related protein (GITR) ligand modulates cytokine release and NK cell reactivity in chronic lymphocytic leukemia (CLL). Leukemia, 26, 991-1000.

Casey SC, Li Y, Felsher DW (2014). An essential role for the immune system in the mechanism of tumor regression following targeted oncogene inactivation. Immunol Res, 58, 282-91.

Etesam Z, Nemati M, Ebrahimizadeh MA, et al (2016). Altered expression of specific transcription factors of Th17 (RORgammat, RORalpha) and Treg Lymphocytes (FOXP3) by peripheral blood mononuclear cells from patients with multiple sclerosis. J Mol Neurosci, 60, 94-101.

Golubovskaya V, Wu L (2016). Different Subsets of T Cells, Memory, Effector Functions, and CAR-T Immunotherapy. Cancers, 8, E36.

Guery L, Hugues S (2015). Th17 cell plasticity and functions in cancer immunity. Biomed Res Int, 2015, 314620.

Hatanaka H, Ishizawa H, Nakamura Y, et al (2014). Effects of vitamin $\mathrm{K} 3$ and $\mathrm{K} 5$ on proliferation, cytokine production, and regulatory $\mathrm{T}$ cell-frequency in human peripheral-blood mononuclear cells. Life Sci, 99, 61-8.

Jaberipour M, Habibagahi M, Hosseini A, et al (2010). Increased CTLA-4 and FOXP3 transcripts in peripheral blood mononuclear cells of patients with breast cancer. Pathol Oncol Res, 16, 547-51.

Jafarzadeh A, Fooladseresht H, Minaee K, et al (2015a). Higher circulating levels of chemokine CCL22 in patients with breast cancer: evaluation of the influences of tumor stage and chemokine gene polymorphism. Tumour Biol, 36, 1163-71.

Jafarzadeh A, Minaee K, Farsinejad AR, et al (2015b). Evaluation of the circulating levels of IL-12 and IL-33 in patients with breast cancer: influences of the tumor stages and cytokine gene polymorphisms. Iran J Basic Med Sci, 18, 1189-98.

Jafarzadeh A, Jamali M, Mahdavi R, et al (2015c). Circulating levels of interleukin-35 in patients with multiple sclerosis: evaluation of the influences of FOXP3 gene polymorphism and treatment program. J Mol Neurosci, 55, 891-7.

Jafarzadeh A, Fooladseresht H, Nemati M, et al (2016). Higher circulating levels of chemokine CXCL10 in patients with breast cancer: Evaluation of the influences of tumor stage and chemokine gene polymorphism. Cancer Biomark, 16, 545-54.

Kawaguchi K, Suzuki E, Yamaguchi A, et al (2017). Altered expression of major immune regulatory molecules in peripheral blood immune cells associated with breast cancer. Breast Cancer, 24, 111-20.

Kawano M, Tanaka K, Itonaga I, et al (2015). Dendritic cells combined with anti-GITR antibody produce antitumor effects in osteosarcoma. Oncol Rep, 34, 1995-2001.

Knee DA, Hewes B, Brogdon JL (2016). Rationale for anti-GITR cancer immunotherapy. Eur J Cancer, 67, 1-10.

Kordi-Tamandani DM, Davani SK, Baranzehi T, Hemati S (2014). Analysis of promoter methylation, polymorphism and expression profile of cytotoxic T-Lymphocyte-associated Antigen-4 in patients with gastric cancer. $J$ Gastrointestin Liver Dis, 23, 249-53.

Kursunel MA, Esendagli G (2016). The untold story of 
IFN-gamma in cancer biology. Cytokine Growth Factor

Rev, 31, 73-81.

Levings MK, Sangregorio R, Sartirana C, et al (2002). Human $\mathrm{CD} 25+\mathrm{CD} 4+\mathrm{T}$ suppressor cell clones produce transforming growth factor beta, but not interleukin 10, and are distinct from type 1 T regulatory cells. $J$ Exp Med, 196, 1335-46.

Lu L, Xu X, Zhang B, et al (2014). Combined PD-1 blockade and GITR triggering induce a potent antitumor immunity in murine cancer models and synergizes with chemotherapeutic drugs. $J$ Transl Med, 12, 36.

Mannino MH, Zhu Z, Xiao H, et al (2015). The paradoxical role of IL-10 in immunity and cancer. Cancer Lett, 367, 103-7.

Monzavi-Karbassi B, Pashov A, Kieber-Emmons T (2013). Tumor-Associated Glycans and Immune Surveillance. Vaccines, 1, 174-203.

Mottaghi A, Salehi E, Sezavar H, et al (2012). The in vitro effect of oxidized LDL and PHA on proliferation and gene expression of regulatory $\mathrm{T}$ cells in patients with atherosclerosis. Iran J Allergy Asthma Immunol, 11, 217-23.

Muenst S, Laubli H, Soysal SD, et al (2016). The immune system and cancer evasion strategies: therapeutic concepts. J Intern Med, 279, 541-62.

Naidoo J, Page DB, Wolchok JD (2014). Immune modulation for cancer therapy. Br J Cancer, 111, 2214-19.

Nasser A, Khosravi A, Taha Gomravi M, Azizian R (2014). The role of T regulatory lymphocytes in lymphoma. Middle East $J$ Cancer, 5, 55-6.

Noack M, Miossec P (2014). Th17 and regulatory T cell balance in autoimmune and inflammatory diseases. Autoimmun Rev, 13, 668-77.

Nocentini G, Ronchetti S, Petrillo MG, Riccardi C (2012). Pharmacological modulation of GITRL/GITR system: therapeutic perspectives. Br J Pharmacol, 165, 2089-99.

Patel MA, Kim JE, Theodros D, et al (2016). Agonist anti-GITR monoclonal antibody and stereotactic radiation induce immune-mediated survival advantage in murine intracranial glioma. J Immunother Cancer, 4, 28.

Rathod S, Das R, Thanapati S, Arankalle V, Tripathy A (2014). Suppressive activity and altered conventional phenotype markers/mediators of regulatory $\mathrm{T}$ cells in patients with self-limiting hepatitis E. J Viral Hepat, 21, 141-51.

Sheikhi A, Jafarzadeh A, Kokhaei P, Hojjat-Farsangi M (2016). Whole tumor cell vaccine adjuvants: Comparing IL-12 to IL-2 and IL-15. Iran J Immunol, 13, 148-66.

Siegel RL, Miller KD, Jemal A (2016). Cancer statistics, 2016. Cancer J Clin, 66, 7-30.

Singletary SE, Allred C, Ashley P, et al (2003). Staging system for breast cancer: revisions for the 6th edition of the AJCC Cancer Staging Manual. Surg Clin North Am, 83, 803-19.

Spranger S (2016). Mechanisms of tumor escape in the context of the T-cell-inflamed and the non-T-cell-inflamed tumor microenvironment. Int Immunol, 28, 383-91.

Szylberg L, Karbownik D, Marszalek A (2016). The role of FOXP3 in human cancers. Anticancer Res, 36, 3789-94.

Takeuchi Y, Nishikawa H (2016). Roles of regulatory T cells in cancer immunity. Int Immunol, 28, 401-9.

Tohyama N, Tanaka S, Onda K, Sugiyama K, Hirano T (2013). Influence of anticancer agents on cell survival, proliferation, and CD4+ CD25+ Foxp3+ regulatory T cell-frequency in human peripheral-blood mononuclear cells activated by $\mathrm{T}$ cell-mitogen. Int Immunopharmacol, 15, 160-6.

\section{(ब) $(\mathbb{8}$}

This work is licensed under a Creative Commons AttributionNon Commercial 4.0 International License. 